\title{
Correction to: Safety and efficacy of microwave versus radiofrequency ablation for large hepatic hemangioma: a multicenter retrospective study with propensity score matching
}

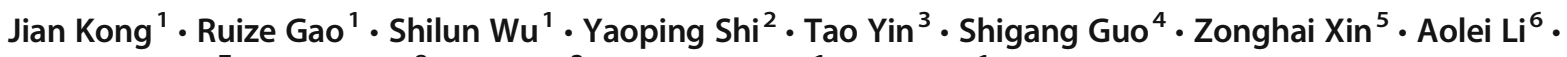 \\ Xinliang Kong ${ }^{7} \cdot$ Demin $_{\mathrm{Ma}^{8}} \cdot$ Bo Zhai $^{2} \cdot$ Wenbing Sun ${ }^{1} \cdot$ Jun Gao ${ }^{1}$
}

Published online: 3 March 2022

(C) The Author(s), under exclusive licence to European Society of Radiology 2022

\section{Correction to: European Radiology}

https://doi.org/10.1007/s00330-021-08425-4

The original version of this article, published on 29 January 2022 unfortunately contained a mistake. The affiliation details of the authors Ruize Gao and Yaoping Shi were incorrectly assigned and have now been corrected as follows.

Ruize Gao: Department of Hepatobiliary Surgery, Beijing Chaoyang Hospital Affiliated To Capital Medical University, Beijing 100,043, China.

Yaoping Shi: Department of Interventional Oncology, Renji Hospital, School of Medicine, Shanghai Jiaotong University, Shanghai 200,120, China.

The original article has been corrected.

Publisher's note Springer Nature remains neutral with regard to jurisdictional claims in published maps and institutional affiliations.

The online version of the original article can be found at https://oi.org/ 10.1007/s00330-021-08425-4

Bo Zhai

zhaiboshi@sina.com

$\triangle$ Wenbing Sun

sunwenbing@bjcyh.com

$\triangle$ Jun Gao

gaojun8430@163.com

1 Department of Hepatobiliary Surgery, Beijing Chaoyang Hospital Affiliated To Capital Medical University, Beijing 100043, China

2 Department of Interventional Oncology, Renji Hospital, School of Medicine, Shanghai Jiaotong University, Shanghai 200120, China

3 Department of General Surgery, Affiliated Hospital of Chifeng University, Chifeng 024000, Inner Mongolia Autonomous Region, China
4 Department of General Surgery, Chaoyang Central Hospital, Chaoyang 122000, Liaoning Province, China

5 Department of General Surgery, Binzhou Second People's Hospital, Binzhou 256000, Shandong Province, China

6 Department of General Surgery, Chaoyang Second Hospital, Chaoyang 122000, Liaoning Province, China

7 Department of Hepatobiliary Surgery, Rizhao Central Hospital, Rizhao 276801, Shandong Province, China

8 Department of Hepatobiliary Surgery, Dezhou People's Hospital, Dezhou 253000, Shandong Province, China 